\title{
Hopf fibration and monopole connection over the contact quantum spheres.
}

\author{
Tomasz Brzeziński \\ Department of Mathematics, University of Wales Swansea \\ Singleton Park, Swansea SA2 8PP, U.K.; T.Brzezinski@swansea.ac.uk \\ Ludwik Dąbrowski \\ Scuola Internazionale Superiore di Studi Avanzati, \\ Via Beirut 2-4, I-34014, Trieste, Italy; dabrow@sissa.it

\begin{abstract}
Bartosz Zieliński
Department of Mathematics, University of Wales Swansea,

and

Department of Theoretical Physics II, University of Eódź

Pomorska 149/153, 90-236 Łódź, Poland.
\end{abstract} \\ Singleton Park, Swansea SA2 8PP, U.K.; mabpz@swan.ac.uk
}

\begin{abstract}
Noncommutative geometry of quantised contact spheres introduced by Omori-MaedaMiyazaki-Yoshioka in [18, 19] is studied. In particular it is proven that these spheres form a noncommutative Hopf fibration in the sense of Hopf-Galois extensions. The monopole (strong) connection is constructed, and projectors describing projective modules of all monopole charges are computed.
\end{abstract}

keywords: Noncommutative geometry, quantum spheres, Hopf fibration, monopole mathclass: Primary 58B34; Secondary 17B37. 


\section{Introduction}

Since its inception in [3], the geometric approach to quantum group gauge theories based on quantum principal bundles or Hopf-Galois extensions, attracted a considerable interest. The general structure of quantum principal bundles and their generalisations known as coalgebra principal bundles or coalgebra-Galois extensions [4, 5] is well-known by now. In particular those Hopf- and coalgebra-Galois extensions which admit a special kind of connections, known as strong connections introduced in [15] seem to be of special interest for non-commutative geometry a la Connes. As recently revealed in [16], 12, [2] the modules of sections of quantum vector bundles associated to such extensions are projective modules, i.e., vector bundles of non-commutative geometry. This makes Galois-type extensions with strong connections perfect candidates for (algebraic) non-commutative principal bundles. For an extensive review of algebraic background and recent progress in this field we refer to 14 .

Despite the fact that the structure of Galois-type extensions is very well-known, it is quite difficult to construct concrete examples of such extensions which in addition admit strong connections. These are technical rather than structural difficulties, hence they do not undermine usefulness of the general theory. On the other hand interest in any abstract theory is fuelled by non-trivial examples. For many years essentially one nontrivial example of a quantum principal bundle with strong connection was known. This was the original example of a quantum Hopf fibration in [3]. Recently, however, a number of new non-trivial examples have been constructed. These include examples obtained by patching of trivial quantum principal bundles [7] as well as examples motivated by recent interest in and several constructions of quantum and non-commutative spheres initiated in [9] and [13] (see [1] for a concise review of low dimensional cases). An earlier deformation of the 3-sphere introduced by Matsumoto in [17] has been shown to give rise to a HopfGalois extension of the (commutative) algebra of functions on the 2-sphere [6]. Other examples, in particular [8] and [1] seem also to fit perfectly into the scheme of Galois-type extensions.

The aim of the present note is to reveal that quantum spheres constructed in [18, 19] as quantisations of contact structures on spherical manifolds, and thus termed contact quantum spheres give rise to quantum principal bundles with strong connections. These are all non-standard deformations of the Hopf fibration. The resulting connections appear to be deformations of the Dirac magnetic monopole field. The general theory guides us then to projectors for the associated quantum line bundles with an arbitrary monopole 
charge.

The paper is ogranised as follows. In Section 2 we describe the non-standard deformation of the 3-sphere, obtained in [18 by the quantisation of the contact structure on the 3 -sphere. We show that this sphere admits an action of the group $U(1)$. In algebraic terms it means that there is a coaction of the algebra of Laurent polynomials in one variable. Next we show that the fixed points of these action (coaction) describe the deformed algebra of the noncommutative 2-sphere, introduced in 18. Finally we show that this structure defines a quantum Hopf fibration (cf. [19, Section 4]), namely that the deformed algebra of the noncommutative 3-sphere is a Hopf-Galois extension of the deformed algebra of the noncommutative 2-sphere. In Section 3 we construct explicitly a strong connection on this Hopf-Galois extension. This immediately gives rise to projectors for quantum associated line bundles, which we explicitly compute. In Section 4 we address some questions about the problem of specifying the formal paramter $\mu$ to a numerical value and about the possibility of a $C^{*}$-algebraic version.

We work over a field of complex numbers $\mathbb{C}$ and unadorned tensor product is over $\mathbb{C}$.

\section{Quantum Hopf fibration}

The algebraic structure underlying quantum group principal bundles is provided by HopfGalois extensions. To construct such an extension one needs the following data. First, let $H$ be a Hopf algebra with coproduct $\Delta: H \rightarrow H \otimes H$, counit $\varepsilon: H \rightarrow \mathbb{C}$ and antipode $S: H \rightarrow H$. Second, let $P$ be a right $H$-comodule algebra, i.e., an algebra and a right $H$-comodule such that the coaction $\Delta_{R}: P \rightarrow P \otimes H$ is an algebra map. For the coaction we use the Sweedler notation $\Delta_{R}(p)=p_{(0)} \otimes p_{(1)}$ (summation understood). With these data one defines the coinvariant subalgebra of $P$ by

$$
B=\left\{x \in P \mid \Delta_{R}(x)=x \otimes 1\right\} .
$$

Since $B$ is a subalgebra of $P$ there is an obvious inclusion map, hence an extension, $B \rightarrow P$. Furthermore, $P$ is a $(B, B)$-bimodule, hence one can consider the tensor product $P \otimes_{B} P$. The extension $B \rightarrow P$ is called a Hopf-Galois extension, provided the canonical left $P$ module, right $H$-comodule map

$$
\text { can }:=\left(m_{P} \otimes \text { id }\right) \circ\left(\text { id } \otimes_{B} \Delta_{R}\right): P \otimes_{B} P \rightarrow P \otimes H,
$$

where $m_{P}$ denotes the product in $P$, is bijective. Explicitly, the map can reads

$$
\operatorname{can}\left(x \otimes_{B} y\right)=x y_{(0)} \otimes y_{(1)} .
$$


The aim of this section is to show that the quantum contact 3-sphere defined in [18] is a Hopf-Galois extension of its algebra of coinvariants. The latter is the algebra of functions of the quantum 2-sphere also defined in [18.

The polynomial *-algebra $A\left(S_{\mu}^{3}\right)$ that underlies the Omori-Maeda-Miyazaki-Yoshioka quantum contact 3 -sphere is generated by a selfadjoint $\mu$ and by $a, b, a^{*}, b^{*}$ with relations

$$
\begin{gathered}
b a=a b, \quad a b^{*}=(1-\mu) b^{*} a, \\
\mu a-a \mu=\mu a \mu, \quad \mu b-b \mu=\mu b \mu, \\
a a^{*}-(1-\mu) a^{*} a=\mu, \quad b b^{*}-(1-\mu) b^{*} b=\mu, \\
a^{*} a+b^{*} b=1 .
\end{gathered}
$$

From the above relations it follows that

$$
a a^{*}+b b^{*}=1+\mu \text {. }
$$

Note also that (1b) is equivalent to

$$
\mu a(1+k \mu)=(1+(k+1) \mu) a \mu, \quad \mu b(1+k \mu)=(1+(k+1) \mu) b \mu
$$

for all $k \in \mathbb{Z}$. We denote by $A^{\prime}\left(S_{\mu}^{3}\right)$ the polynomial $*$-algebra $A\left(S_{\mu}^{3}\right)$ with the generator $\mu$ required to be invertible (i.e. with adjoined $\mu^{-1}$ ).

It turns out convenient for our purposes to employ a certain $\mu$-regulated smooth algebra $A^{\infty}\left(S_{\mu}^{3}\right)$, which is defined and studied in [18. The algebra $A^{\infty}\left(S_{\mu}^{3}\right)$, called noncommutative contact algebra on $S^{3}$, contains densely the polynomial *-algebra $A^{\prime}\left(S_{\mu}^{3}\right)$. Also, it contains $f(\mu)$ for any formal power series $f$, and the following relations are fulfilled

$$
\begin{aligned}
a f(\mu) & =f\left(\frac{\mu}{1+\mu}\right) a, & f(\mu) a & =a f\left(\frac{\mu}{1-\mu}\right), \\
b f(\mu) & =f\left(\frac{\mu}{1+\mu}\right) b, & f(\mu) b & =b f\left(\frac{\mu}{1-\mu}\right) .
\end{aligned}
$$

In particular, for all $k \in \mathbb{Z}$ the elements $1+k \mu$ are invertible and have square root in $A^{\infty}\left(S_{\mu}^{3}\right)$. In the sequel we shall need their inverses as well as the square roots, which satisfy the following relations $\forall k \in \mathbb{Z}$,

$$
a \mu(1+k \mu)^{-1}=\mu(1+(k+1) \mu)^{-1} a, \quad b \mu(1+k \mu)^{-1}=\mu(1+(k+1) \mu)^{-1} b
$$

and

$$
a \sqrt{1+k \mu}=\frac{\sqrt{1+(k+1) \mu}}{\sqrt{1+\mu}} a, \quad b \sqrt{1+k \mu}=\frac{\sqrt{1+(k+1) \mu}}{\sqrt{1+\mu}} b .
$$


Although $\mu$ is a generator it can be regarded as a noncentral formal parameter, cf. [18] for a precise meaning of this statement. Also, from the defining relations of (10) it is apparent that $A^{\infty}\left(S_{\mu}^{3}\right)$ is a $\mathbb{Z}$-graded algebra with the grading defined by setting

$$
\operatorname{deg}(a)=\operatorname{deg}(b)=1, \operatorname{deg}\left(a^{*}\right)=\operatorname{deg}\left(b^{*}\right)=-1, \operatorname{deg}(\mu)=0 .
$$

This in turn allows us to view $A^{\infty}\left(S_{\mu}^{3}\right)$ as a comodule algebra of the Hopf algebra $H$ of functions on $U(1)$. Explicitly, $H=\mathbb{C}\left[u, u^{-1}\right]$ is an algebra of Laurent polynomials in one variable $u$ (i.e., $u^{-1}$ is the multiplicative inverse of $u$ ), and it is a *-algebra with $u^{*}=u^{-1}$. A Hopf algebra structure of $H$ is determined by $\Delta(u)=u \otimes u, \varepsilon(u)=1$ and $S(u)=u^{-1}$. The grading of $A^{\infty}\left(S_{\mu}^{3}\right)$ makes it a right comodule algebra with the coaction given on homogeneous elements by

$$
\Delta_{R}(x)=x \otimes u^{\operatorname{deg}(x)} .
$$

Thus explicitly on generators the coaction comes out as

$$
\Delta_{R}(a)=a \otimes u, \Delta_{R}(b)=b \otimes u, \Delta_{R}\left(a^{*}\right)=a^{*} \otimes u^{-1}, \Delta_{P}\left(b^{*}\right)=b^{*} \otimes u^{-1}, \Delta_{P}(\mu)=\mu \otimes 1 .
$$

Note that the coaction $\Delta_{R}$ is compatible with the $*$-structure (it is a $*$-algebra homomorphism).

The definition of the coaction in terms of the grading immediately implies that the coinvariant subalgebra coincides with the zero-degree subalgebra, i.e.,

$$
A^{\infty}\left(S_{\mu}^{2}\right):=\left\{x \in A^{\infty}\left(S_{\mu}^{3}\right) \mid \Delta_{R}(x)=x \otimes 1\right\}=\left\{x \in A^{\infty}\left(S_{\mu}^{3}\right) \mid \operatorname{deg}(x)=0\right\} .
$$

Using equation (4) it is immediate to verify that $\mu$ is central element in $A^{\infty}\left(S_{\mu}^{2}\right)$. It can be also seen that $A^{\infty}\left(S_{\mu}^{2}\right)$ is the commutant of $\mu$ in $A^{\infty}\left(S_{\mu}^{3}\right)$.

The relations (11), (41) and (5) provide us with a deeper insight into the structure of $A^{\infty}\left(S_{\mu}^{2}\right)$. With their help we can establish that $A^{\infty}\left(S_{\mu}^{2}\right)$ contains a (dense) polynomial *-algebra $A^{\prime}\left(S_{\mu}^{2}\right)$ generated by

$$
X=X^{*}=a a^{*}-(\mu+1) / 2, \quad Z=a b^{*}, \quad Z^{*}=b a^{*},
$$

and self-adjoint (invertible) element $\mu$. Notice that $A^{\prime}\left(S_{\mu}^{2}\right)$ is contained strictly in the commutant of $\mu$ in $A^{\prime}\left(S_{\mu}^{3}\right)$, which coincides with the grade zero subalgebra of $A^{\prime}\left(S_{\mu}^{3}\right)$ and also with the $H$-coinvariant subalgebra of $A^{\prime}\left(S_{\mu}^{3}\right)$. Similar observations hold for $A\left(S_{\mu}^{2}\right)$, defined as the $*$-algebra obtained from $A^{\prime}\left(S_{\mu}^{2}\right)$ by omitting the invertibility of $\mu$. 
The relations in $A^{\infty}\left(S_{\mu}^{2}\right)$ are derived from the relations in $A^{\infty}\left(S_{\mu}^{3}\right)$ and come out as

$$
\begin{gathered}
\mu X-X \mu=0, \mu Z-Z \mu=0, \\
X Z-Z X=-\mu Z, \\
Z Z^{*}-Z^{*} Z=-2 \mu X, \\
\left(X+\frac{\mu}{2}\right)^{2}+Z Z^{*}=\frac{1}{4},
\end{gathered}
$$

Note that from the relations above it follows that there is also a second radial relation

$$
\left(X-\frac{\mu}{2}\right)^{2}+Z^{*} Z=\frac{1}{4} .
$$

Since $\mu$ is central, it could be possible to consider $\mu$ as a formal parameter and specify it to a numerical value, we shall comment on this issue in the final section.

We claim that in a 'dual' sense the quantum contact three sphere $S_{\mu}^{3}$ is a total space of a quantum $U(1)$-principal bundle over the quantum two-sphere $S_{\mu}^{2}$, i.e., $A^{\infty}\left(S_{\mu}^{3}\right)$ is a Hopf-Galois extension of $A^{\infty}\left(S_{\mu}^{2}\right)$ with the structure Hopf algebra $H$. This claim is proven by explicit construction of the inverse to the canonical map.

To relieve the notation we write $P$ for $A^{\infty}\left(S_{\mu}^{3}\right)$ and $B$ for $A^{\infty}\left(S_{\mu}^{2}\right)$. Consider the map can $^{-1}: P \otimes H \rightarrow P \otimes_{B} P$ defined for all $x \in P$ and $n \in \mathbb{N}$ by

$$
\begin{aligned}
\operatorname{can}^{-1}\left(x \otimes u^{n}\right) & =\sum_{k=0}^{n}\left(\begin{array}{l}
n \\
k
\end{array}\right) x\left(a^{*}\right)^{n-k}\left(b^{*}\right)^{k} \otimes_{B} b^{k} a^{n-k}, \\
\operatorname{can}^{-1}\left(x \otimes u^{-n}\right) & =x(1+n \mu)^{-1} \sum_{k=0}^{n}\left(\begin{array}{l}
n \\
k
\end{array}\right) a^{n-k} b^{k} \otimes_{B}\left(b^{*}\right)^{k}\left(a^{*}\right)^{n-k},
\end{aligned}
$$

where $\left(\begin{array}{l}n \\ k\end{array}\right)$ are the usual binomial coefficients. Directly from the definition it follows that $\mathrm{can}^{-1}$ is a left $P$-module map. Furthermore, the degree counting on the right hand side and the comparison of the powers of $u$ immediately confirm that $\operatorname{can}^{-1}$ is a right $H$-comodule map. Before we prove that $\operatorname{can}^{-1}$ is the inverse map to can we note that for all $n \in \mathbb{N}$,

$$
\begin{aligned}
& \sum_{k=0}^{n}\left(\begin{array}{l}
n \\
k
\end{array}\right)\left(a^{*}\right)^{n-k}\left(b^{*}\right)^{k} b^{k} a^{n-k}=1, \\
& \sum_{k=0}^{n}\left(\begin{array}{l}
n \\
k
\end{array}\right) a^{n-k} b^{k}\left(b^{*}\right)^{k}\left(a^{*}\right)^{n-k}=1+n \mu .
\end{aligned}
$$

The formulae (8) are most easily proven by induction. They are clearly satisfied for $n=1$. Next, assume that they hold for $n-1$ with $n \geq 2$. Using equations (1d), (2) and the 
well-known formula

$$
\sum_{l=0}^{k}(-1)^{l}\left(\begin{array}{c}
n \\
k-l
\end{array}\right)=\left(\begin{array}{c}
n-1 \\
k
\end{array}\right)
$$

observe that

$$
\begin{gathered}
\sum_{k=0}^{n}\left(\begin{array}{l}
n \\
k
\end{array}\right)\left(a^{*}\right)^{n-k}\left(b^{*}\right)^{k} b^{k} a^{n-k}=\sum_{k=0}^{n-1}\left(\begin{array}{c}
n-1 \\
k
\end{array}\right)\left(a^{*}\right)^{n-1-k}\left(b^{*}\right)^{k} b^{k} a^{n-1-k}, \\
\sum_{k=0}^{n}\left(\begin{array}{l}
n \\
k
\end{array}\right) a^{n-k} b^{k}\left(b^{*}\right)^{k}\left(a^{*}\right)^{n-k}=\sum_{k=0}^{n-1}\left(\begin{array}{c}
n-1 \\
k
\end{array}\right) a^{n-1-k} b^{k}(1+\mu)\left(b^{*}\right)^{k}\left(a^{*}\right)^{n-1-k} .
\end{gathered}
$$

Then using (44) we conclude that equations (8a, 8b) hold for all $n$. Now we are in position to prove that $\mathrm{can}^{-1}$ is the inverse of can. Take $x \in P$ and $n \in \mathbb{N}$. Then

$$
\begin{aligned}
\operatorname{can}\left(\operatorname{can}^{-1}\left(x \otimes u^{n}\right)\right) & =\operatorname{can}\left(\sum_{k=0}^{n}\left(\begin{array}{l}
n \\
k
\end{array}\right) x\left(a^{*}\right)^{n-k}\left(b^{*}\right)^{k} \otimes_{B} b^{k} a^{n-k}\right) \\
& =\sum_{k=0}^{n}\left(\begin{array}{l}
n \\
k
\end{array}\right) x\left(a^{*}\right)^{n-k}\left(b^{*}\right)^{k} b^{k} a^{n-k} \otimes u^{n}=x \otimes u^{n},
\end{aligned}
$$

where the last equality follows from (8a $)$. Similarly, the use of (8b) confirms that

$$
\operatorname{can}\left(\operatorname{can}^{-1}\left(x \otimes u^{-n}\right)\right)=x \otimes u^{-n}
$$

Conversely we need to check the equality $\operatorname{can}^{-1}\left(\operatorname{can}\left(x \otimes_{B} y\right)\right)=x \otimes_{B} y$ for all $x, y \in P$. Since $P$ is a $\mathbb{Z}$-graded algebra suffices it to take homogeneous $y$ of degree $n$. Suppose $n \geq 0$. Then

$$
\operatorname{can}^{-1}\left(\left(\operatorname{can}\left(x \otimes_{B} y\right)\right)=\operatorname{can}^{-1}\left(x y \otimes u^{n}\right)=\sum_{k=0}^{n}\left(\begin{array}{l}
n \\
k
\end{array}\right) x y\left(a^{*}\right)^{n-k}\left(b^{*}\right)^{k} \otimes_{B} b^{k} a^{n-k} .\right.
$$

Since $\operatorname{deg}(y)=n$, each of the $y\left(a^{*}\right)^{n-k}\left(b^{*}\right)^{k}$ has degree 0 , hence it is in $B$ and we can write

$$
\operatorname{can}^{-1}\left(\left(\operatorname{can}\left(x \otimes_{B} y\right)\right)=\sum_{k=0}^{n}\left(\begin{array}{l}
n \\
k
\end{array}\right) x \otimes_{B} y\left(a^{*}\right)^{n-k}\left(b^{*}\right)^{k} b^{k} a^{n-k}=x \otimes_{B} y,\right.
$$

by (8a ). In the case of homogeneous $y$ of negative degree, we use equation (8b) to obtain the assertion. Thus we have proven that can is a bijective map, i.e., $A^{\infty}\left(S_{\mu}^{3}\right)$ is a Hopf-Galois extension of $A^{\infty}\left(S_{\mu}^{2}\right)$ as claimed. 


\section{Monopole connection and projectors of charge $n$}

\subsection{Strong connection}

Connections in quantum principal bundles are defined as colinear splittings of one forms into horizontal and vertical parts (cf. [3]). From the non-commutative geometry point of view a special class of connections, introduced in [15] and known as strong connections, is of particular interest.

Algebraically, one-forms on an algebra $P$ are defined as a $P$-bimodule $\Omega^{1}(P)$ together with a linear map $\mathrm{d}: P \rightarrow \Omega^{1}(P)$ which satisfies the Leibniz rule $\mathrm{d}\left(p p^{\prime}\right)=\mathrm{d}(p) p^{\prime}+p \mathrm{~d}\left(p^{\prime}\right)$. In what follows we restrict ourselves to the particular example of differential forms, known as universal differential forms and denoted by $\Omega^{1} P$. The bimodule $\Omega^{1} P$ is defined as the kernel of the multiplication map $m_{P}$, and the differential in this case is given by the map

$$
\mathrm{d}: P \rightarrow \Omega^{1} P=\operatorname{ker} m_{P}, \quad p \mapsto 1 \otimes p-p \otimes 1 .
$$

In the universal differential calculus case, strong connections on a Hopf-Galois extension $B \rightarrow P$ are in one-to-one correspondence with strong connection one forms. These are defined as homomorphisms $\omega: H \rightarrow \Omega^{1} P$ vanishing on 1 and satisfying the following three conditions (see [12, Theorem 2.3] for various equivalent descriptions of strong connections):

$$
\begin{gathered}
\Delta_{\Omega^{1} P} \circ \omega=(\omega \otimes \mathrm{id}) \circ \mathrm{Ad}, \\
\left(m_{P} \otimes \mathrm{id}\right) \circ\left(\mathrm{id} \otimes \Delta_{R}\right) \circ \omega=1 \otimes(\mathrm{id}-\varepsilon), \\
\mathrm{d} p-p_{(0)} \omega\left(p_{(1)}\right) \in\left(\Omega^{1} B\right) P, \quad \forall p \in P .
\end{gathered}
$$

A few of the symbols above require explanation. The map $\Delta_{\Omega^{1} P}$ on the left hand side of equation (10a) is the right caction of $H$ on $\Omega^{1} P$ obtained be restricting the right coaction of $H$ on the tensor product $P \otimes P$ (right diagonal coaction). Explicitly,

$$
\Delta_{\Omega^{1} P}: \Omega^{1} P \rightarrow \Omega^{1} P \otimes H, \quad \sum_{i} p^{i} \otimes \tilde{p}^{i} \mapsto \sum_{i} p_{(0)}^{i} \otimes \tilde{p}_{(0)}^{i} \otimes p_{(1)}^{i} \tilde{p}_{(1)}^{i} .
$$

The map Ad on the right hand side of equation (10a) is the right adjoint coaction of $H$ on itself, i.e., Ad : $H \rightarrow H \otimes H, h \mapsto h_{(2)} \otimes S\left(h_{(1)}\right) h_{(3)}$. Thus equation (10a) corresponds to the classical ad-covariance property of a connection one-form. The map

$$
\chi=\left(m_{P} \otimes \mathrm{id}\right) \circ\left(\mathrm{id} \otimes \Delta_{R}\right)
$$


on the left hand side of equation (10b) has a geometric meaning of a horizontal lift, i.e., an operation which lifts an element of the Lie algebra of a structure group of a principal bundle to a vector tangent to a fibre. Thus equation (10b) has the classical geometric meaning of the property that a connection form evaluated at a horizontal lift of an element of a Lie algebra, returns back this element. Finally, equation (10c) is the strongness condition, which distinguishes strong connections within a class of all connections on a Hopf-Galois extension.

Suppose that the antipode $S \in H$ is invertible. In this case $P$ is a left $H$-comodule with the coaction

$$
\Delta_{L}: P \rightarrow H \otimes P, \quad p \mapsto S^{-1} p_{(1)} \otimes p_{(0)} .
$$

One then proves that if there is a map $\ell: H \rightarrow P \otimes P$ which satisfies the following conditions

$$
\begin{gathered}
\ell(1)=1 \otimes 1, \\
\chi(\ell(h))=1 \otimes h, \\
\ell\left(h_{(1)}\right) \otimes h_{(2)}=\left(\mathrm{id} \otimes \Delta_{R}\right) \circ \ell(h), \\
h_{(1)} \otimes \ell\left(h_{(2)}\right)=\left(\Delta_{L} \otimes \mathrm{id}\right) \circ \ell(h),
\end{gathered}
$$

for all $h \in H$, then the map

$$
\omega: H \rightarrow \Omega^{1} P, \quad h \mapsto \ell(h)-\varepsilon(h) 1 \otimes 1
$$

is a strong connection form. This is, in fact, an equivalent description of strong connection one forms (cf. 2] for the proof and discussion in more general situation). Note that equations (11c)-(11d) simply state that $\ell$ is an $H$-bicomodule map, where $H$ is a viewed as a bicomodule via the coproduct, and $P \otimes P$ is an $H$-bicomodule with coactions $\Delta_{L} \otimes P$ and $P \otimes \Delta_{R}$.

The antipode of the Hopf algebra $H$ of functions on $U(1)$ is involutive, i.e. $S \circ S=$ id, hence, in particular, invertible. Thus we can define the left coaction $\Delta_{L}$ for $P=A^{\infty}\left(S_{\mu}^{3}\right)$. This again is defined in terms of the $\mathbb{Z}$-grading and comes out as

$$
\Delta_{L}(x)=u^{-\operatorname{deg}(x)} \otimes x
$$

for any homogeneous $x \in P$. Thus we can follow the above procedure in the case of a 
Hopf-Galois extension $P=A^{\infty}\left(S_{\mu}^{3}\right)$ of $B=A^{\infty}\left(S_{\mu}^{2}\right)$, and define $\ell: H \rightarrow P \otimes P$ by

$$
\begin{aligned}
\ell\left(u^{n}\right) & =\sum_{k=0}^{n}\left(\begin{array}{l}
n \\
k
\end{array}\right)\left(a^{*}\right)^{n-k}\left(b^{*}\right)^{k} \otimes b^{k} a^{n-k}, \\
\ell\left(u^{-n}\right) & =(1+n \mu)^{-1} \sum_{k=0}^{n}\left(\begin{array}{l}
n \\
k
\end{array}\right) a^{n-k} b^{k} \otimes\left(b^{*}\right)^{k}\left(a^{*}\right)^{n-k}, \\
\ell(1) & =1 \otimes 1,
\end{aligned}
$$

for $n \in \mathbb{N}$. Note that this is simply the expression for $\operatorname{can}^{-1}(1 \otimes h)$ lifted to $P \otimes P$ by omitting the decoration ${ }_{B}$ on $\otimes_{B}$ (cf. equation (17)). By definition of $\ell$ the equation (11a) is satisfied. Next, since $\chi$ is a lifitng of the canonical map can to $P \otimes P$, similar arguments to those used to prove that $\mathrm{can}^{-1}$ is the right inverse of can ensure that equation (11b) is satisfied. Moreover, similarly as in the discussion after the definition of can $^{-1}$ by counting the degree it follows that $\ell$ is a right $H$-colinear map, hence equation (11c) holds. Finally since $\operatorname{deg}\left(\left(a^{*}\right)^{n-k}\left(b^{*}\right)^{k}\right)=-n$ and $\operatorname{deg}\left(a^{n-k} b^{k}\right)=n$ one easily realises that the map $\ell$ is also left $H$-colinear, so that equation (11d) is satisfied. Thus we have constructed a strong connection in the quantum contact Hopf fibration with the strong connection form $\omega\left(u^{n}\right)=\ell\left(u^{n}\right)-1 \otimes 1$, for all $n \in \mathbb{Z}$.

\section{$3.2 \quad$ Projectors}

To any Hopf-Galois extension $B \rightarrow P$ with the structure Hopf algebra $H$ and any right $H$ comodule $V$ one can associate a left $B$-module $\Gamma^{H}(V, P)$ which plays the role of the module of sections of the associated quantum vector bundle. Explicilty, $\Gamma^{H}(V, P)$ is a vector space of right $H$-comodule maps $\phi: V \rightarrow P$ with the $B$-action given by $(b \cdot \phi)(v)=b \phi(v)$. If a Hopf-Galois extension $B \rightarrow P$ admits a strong connection and $H$ has a bijective antipode then for any finite-dimensional $V, \Gamma^{H}(V, P)$ is a finitely generated projective left $B$-module (cf. [12]). Furthermore, the covariant derivative corresponding to a strong connection gives rise to a connection in module $\Gamma^{H}(V, P)$.

For any map $\ell: H \rightarrow P \otimes P$ satisfying conditions (11) write for all $h \in H$,

$$
\ell(h)=\sum_{i} \ell_{i}^{[1]}(h) \otimes \ell_{i}^{[2]}(h) .
$$

Although the number $r$ of terms in the sum on the right hand side may depend on $h$, it is always finite. Application of id $\otimes \varepsilon$ to both sides of equation (11b) yields

$$
\sum_{i} \ell_{i}^{[1]}(h) \ell_{i}^{[2]}(h)=\varepsilon(h)
$$


Since the left hand side of equation (15) is finite, for any $h \in H$ one can define a square matrix $p(h)$ by

$$
P \ni p(h)_{i j}=\ell_{i}^{[2]}(h) \ell_{j}^{[1]}(h) .
$$

Equation (15) immediately implies that $\sum_{k} p(h)_{i k} p(h)_{k j}=\varepsilon(h) p(h)_{i j}$, hence the matrix $p(h)$ is an idempotent, provided $\varepsilon(h)=1$. Furthermore, if $h$ is a grouplike element, i.e., $\Delta(h)=h \otimes h$, then equations (11c), (11d) imply that $\Delta_{R}\left(p(h)_{i j}\right)=p(h)_{i j} \otimes 1$, i.e., $p(h)_{i j} \in B$. In other words, for any grouplike element $h \in H, p(h)$ is an $r \times r$ matrix ( $r$ being the number of terms in (14) $)$, which is an idempotent in a matrix ring over $B$. Therefore it defines a finitely generated projective module via $B^{r} p(h)$. The connection corresponding to $\ell$ is simply the Grassmann or Levi-Civita connection in $B^{r} p(h)$, i.e., a connection determined by the idempotent (cf. [10]).

In the case of the Hopf-Galois extension $A^{\infty}\left(S_{\mu}^{2}\right) \rightarrow A^{\infty}\left(S_{\mu}^{3}\right)$ each of the $u^{n}$ is a grouplike element. Therefore the map $\ell$ defined by formulae (13) gives an infinite family of idempotents $p\left(u^{n}\right), n \in \mathbb{Z}$. Each of the $p\left(u^{n}\right)$ is an $(n+1) \times(n+1)$-matrix with entries from $A^{\infty}\left(S_{\mu}^{2}\right)$ (the latter claim can be easily confirmed by the degree counting). Obviously there is an ambiguity in factorising $\ell$ into $\ell^{[1]} \otimes \ell^{[2]}$ (scalar coefficients can be factorised in infinitely many ways into legs of tensor product). However if one requires $p\left(u^{n}\right)$ to be Hermitian (i.e. projectors in $B$ ) then the unique possibility turns out to be

$$
\begin{aligned}
\ell(1) & =1 \otimes 1 \\
\ell\left(u^{n}\right) & =\sum_{k=0}^{n}\left[\sqrt{\left(\begin{array}{l}
n \\
k
\end{array}\right)}\left(a^{*}\right)^{n-k}\left(b^{*}\right)^{k}\right] \otimes\left[\sqrt{\left(\begin{array}{l}
n \\
k
\end{array}\right)} b^{k} a^{n-k}\right], \\
\ell\left(u^{-n}\right) & =\sum_{k=0}^{n}\left[\sqrt{\left(\begin{array}{l}
n \\
k
\end{array}\right)}(1+n \mu)^{-1} a^{n-k} b^{k}\right] \otimes\left[\sqrt{\left(\begin{array}{l}
n \\
k
\end{array}\right)}\left(b^{*}\right)^{k}\left(a^{*}\right)^{n-k}\right],
\end{aligned}
$$

for $n \in \mathbb{N}$. This choice leads to an infinite family of Hermitian projectors with entries from $A^{\infty}\left(S_{\mu}^{2}\right)$. Explicitly, $p(1)=1$, and

$$
\begin{aligned}
p\left(u^{n}\right)_{k l} & =\sqrt{\left(\begin{array}{l}
n \\
k
\end{array}\right)\left(\begin{array}{l}
n \\
l
\end{array}\right)} b^{k} a^{n-k}\left(a^{*}\right)^{n-l}\left(b^{*}\right)^{l}, \\
p\left(u^{-n}\right)_{k l} & =\sqrt{\left(\begin{array}{l}
n \\
k
\end{array}\right)\left(\begin{array}{l}
n \\
l
\end{array}\right)}\left(b^{*}\right)^{k}\left(a^{*}\right)^{n-k}(1+n \mu)^{-1} a^{n-l} b^{l},
\end{aligned}
$$

$n \in \mathbb{N}$. At this point it is interesting to mention that apparently these fromulae are polynomial in $a, b, a^{*}, b^{*}$, and polynomial only in $(1+n \mu)^{-1}$, with $n \in \mathbb{N}$, but not in $\mu$ (e.g. (18b) ). However, their entries have to be properly rearranged in order to express 
them in terms of the generators $X, Z, Z^{*}$. In $A^{\infty}\left(S_{\mu}^{3}\right)$ this can be always done with the help of relations (11) and (4) at the cost of creating new expressions $(1+n \mu)^{-1}$ in (18a) and $(1-n \mu)^{-1}$ in $(18 \mathrm{~b})$, with $n \in \mathbb{N}$. Alltogether the whole set of projectors can be rewritten in terms of $X, Z, Z^{*}$ and all $(1+k \mu)^{-1}$ with $k \in \mathbb{Z}$. (The reason for this behaviour is that the the elements $X, Z, Z^{*}$ and $\mu$ do not generate the whole grade-zero polynomial subalgebra of $A\left(S_{\mu}^{3}\right)$.) For instance the first few projectors $p(u), p\left(u^{-1}\right), p\left(u^{2}\right), p\left(u^{-2}\right)$ come out in a matrix form as

$$
\begin{aligned}
& p(u)=\left(\begin{array}{cc}
\frac{1}{2}(1+\mu)+X & Z \\
Z^{*} & \frac{1}{2}(1+\mu)-X
\end{array}\right), \\
& p\left(u^{-1}\right)=\left(\begin{array}{cc}
\frac{1}{2}(1-\mu)+X & Z^{*} \\
Z & \frac{1}{2}(1-\mu)-X
\end{array}\right), \\
& p\left(u^{2}\right)=\frac{1}{1+\mu} \\
& \times\left(\begin{array}{ccc}
\left(X+\frac{1+\mu}{2}\right)\left(X+\frac{1+3 \mu}{2}\right) & \sqrt{2}\left(X+\frac{1+3 \mu}{2}\right) Z & Z^{2} \\
\sqrt{2} Z^{*}\left(X+\frac{1+3 \mu}{2}\right) & 2\left(\frac{1+\mu}{2}+X\right)\left(\frac{1+\mu}{2}-X\right) & \sqrt{2}\left(\frac{1+\mu}{2}-X\right) Z \\
\left(Z^{*}\right)^{2} & \sqrt{2} Z^{*}\left(\frac{1+\mu}{2}-X\right) & \left(\frac{1+\mu}{2}-X\right)\left(\frac{1+3 \mu}{2}-X\right)
\end{array}\right), \\
& p\left(u^{-2}\right)=\frac{1}{1-\mu} \\
& \times\left(\begin{array}{ccc}
\left(X+\frac{1-\mu}{2}\right)\left(X+\frac{1-3 \mu}{2}\right) & \sqrt{2}\left(X+\frac{1-3 \mu}{2}\right) Z^{*} & \left(Z^{*}\right)^{2} \\
\sqrt{2} Z\left(X+\frac{1-3 \mu}{2}\right) & 2\left(\frac{1-\mu}{2}+X\right)\left(\frac{1-\mu}{2}-X\right) & \sqrt{2}\left(\frac{1-\mu}{2}-X\right) Z^{*} \\
Z^{2} & \sqrt{2} Z\left(\frac{1-\mu}{2}-X\right) & \left(\frac{1-\mu}{2}-X\right)\left(\frac{1-3 \mu}{2}-X\right)
\end{array}\right) .
\end{aligned}
$$

Note an interesting symmetry between $p\left(u^{n}\right)$, and $p\left(u^{-n}\right)$ for low values of $n$. The projector $p\left(u^{-n}\right)$ is obtained from the projector $p\left(u^{n}\right)$ by replacing $\mu$ by $-\mu$ and interchanging of $Z$ with $Z^{*}$. This is true for any value of charge $n$ as can be verified directly from the explicit expressions for greater charges $n$, which can be presented. However this follows also from the following symmetry properties. First observe that the transformation

$$
Z \mapsto Z^{*}, \quad Z^{*} \mapsto Z, \quad X \mapsto X, \quad \mu \mapsto-\mu
$$

does not affect the defining relations (6) and defines an automorphism of the algebra $A^{\infty}\left(S_{\mu}^{2}\right)$. This symmetry of $A^{\infty}\left(S_{\mu}^{2}\right)$ comes in fact from the following symmetry of $A^{\infty}\left(S_{\mu}^{3}\right)$. 
Using the elements $\sqrt{1+k \mu} \in A^{\infty}\left(S_{\mu}^{3}\right)$, their inverses $1 / \sqrt{1+k \mu}$ for $k \in \mathbb{Z}$ and the relations (5) we see that the map

$$
\vartheta: A^{\infty}\left(S_{\mu}^{3}\right) \rightarrow A^{\infty}\left(S_{\mu}^{3}\right), \quad \mu \mapsto-\mu, \quad a \mapsto A=\sqrt{1-\mu} a^{*}, \quad b \mapsto B=\sqrt{1-\mu} b^{*}
$$

extends to an algebra automorphism. Note that $\vartheta$ maps degree $n$ elements to degree $-n$ elements, and on the level of degree 0 elements corresponds to the automorphism of $A^{\infty}\left(S_{\mu}^{2}\right)$ given by (20). Now, using the relations

$$
a^{n}(1-\mu)=\frac{1+(n-1) \mu}{1+n \mu} a^{n}, \quad b^{n}(1-\mu)=\frac{1+(n-1) \mu}{1+n \mu} b^{n}, \quad n>0
$$

and the fact that $\mu$ is central in $A^{\infty}\left(S_{\mu}^{2}\right)$ we find that

$$
\vartheta\left(p\left(u^{n}\right)_{k l}\right)=p\left(u^{-n}\right)_{k l} \text {. }
$$

Thus the authomorphism $\left.\vartheta\right|_{A^{\infty}\left(S_{\mu}^{2}\right)}: A^{\infty}\left(S_{\mu}^{2}\right) \rightarrow A^{\infty}\left(S_{\mu}^{2}\right)$ turns the degree $n$ projectors into degree $-n$ projectors, and its existence proves the stated symmetry of monopole projectors.

\section{Final remarks}

If $\mu$ is not required to be invertibile the underlying polynomial *-algebra $A\left(S_{\mu}^{3}\right)$ admits a specification to $\mu=0$, after which it coincides with the usual $*$-algebra of polynomials on the 'classical' $S^{3}$. As far as the polynomial $*$-algebra $A^{\prime}\left(S_{\mu}^{2}\right)$ is concerned since $\mu$ is central it can be specified to any nonzero real value. This yields a family of quantum 2-spheres isomorphic to the universal enveloping algebra of $s u(2)$ with a constrained value $1 / \mu^{2}$ of the quadratic Casimir element (c.f. [11]). The *-algebra $A\left(S_{\mu}^{2}\right)$ (i.e., when the invertibility of $\mu$ is not assumed) admits in addition a specification to $\mu=0$, which clearly corresponds to polynomials on the 'classical' $S^{2}$. Note that the equations (18) with $\mu$ considered as a real deformation parameter rather than as a central generator define also a family of projectors $p\left(u^{n}\right)$ over such quantum 2-spheres. In particular, when $\mu=0$, the projectors $p\left(u^{n}\right)$ correspond to line bundle projectors of the monopole charge or Chern's number $n$ over $S^{2}$. Note also that then $\vartheta$ defined by (21) is the orientation reversing automorphism of $S^{3}$.

It would be interesting to investigate further our bundles and the associated projective modules, especially the Chern-Connes pairing between $K$-theory and $K$-homology. For that aim it would be convenient to have a $C^{*}$-algebraic version of the construction above as otherwise computation of e.g. the $K$-groups is a formidable task. It can be seen that 
the *-algebras $A\left(S_{\mu}^{3}\right)$ and $A\left(S_{\mu}^{2}\right)$ admit certain $C^{*}$-algebraic completions. Although intuitively resembling some topological quantum four (resp. three) dimensional spaces rather than 3-spheres (resp. 2-spheres), they nevertheless would constitute interesting examples. Unfortunately, there is one obstacle for this task. In order to write our formulae (17) for the inverse of the canonical map and then for the connection and the projectors we should adjoin to $A\left(S_{\mu}^{3}\right)$ and to $A\left(S_{\mu}^{2}\right)$ an infinite number of elements $(1+k \mu)^{-1}, k \in \mathbb{Z}$. (To be able to implement the symmetry discussed at the end of Section 3.2 we should adjoin additionally the elements $\sqrt{1+k \mu}$ and $1 / \sqrt{1+k \mu}$.) It can be seen that this spoils not only the $C^{*}$-algebraic completion but even the $*$-algebraic version. This follows from the representation theory of e.g. $A\left(S_{\mu}^{2}\right)$, which can be inferred from that of $s u(2)$. In fact all bounded representations decompose into finite dimensional irreducible ones. These in turn are as follows. There is a family of one-dimensional representations (characters) parametrised by the points of $S^{2}$, which represent $\mu$ by 0 (they obviously do not extend to $A^{\prime}\left(S_{\mu}^{2}\right)$ ). In addition, in each dimension $N \in \mathbb{N}$ there are two $*$-representations, labeled by $\sigma= \pm 1$, which represent $\mu$ either by $1 / N$ or by $-1 / N$. Namely, they are given by

$$
\begin{aligned}
& \mu v_{m}=\frac{\sigma}{N} v_{m}, \\
& X v_{m}=\frac{\sigma m}{N} v_{m}, \\
& Z v_{m}=\frac{\sigma}{2 N} \sqrt{(N+1-2 m)(N-1+2 m)} v_{m-1}, \\
& Z^{*} v_{m}=\frac{\sigma}{2 N} \sqrt{(N-1-2 m)(N+1+2 m)} v_{m+1},
\end{aligned}
$$

with respect to an orthonormal basis $v_{m}$, where $m \in\left\{-\frac{N-1}{2},-\frac{N-3}{2}, \ldots, \frac{N-3}{2}, \frac{N-1}{2}\right\}$.

This excludes a possibility of adjoining the needed elements if we want our $C^{*}$-algebra to describe more than merely the commutative $S^{2}$. At this point it is interesting to make the following observation. Had we constrained ourselves to construct just certain subclass of projectors, say for a selected class of charges, we might have a chance to accomplish a nondegenerate $*$-algebra extended by some (but not all) elements $(1+k \mu)^{-1}$ and then also its $C^{*}$-algebraic version. For instance restricting only to the positive charges $n$ leaves at our disposal the series of all representations that represent $\mu$ by $1 / N$. It is not clear however what could be a possible interpretation of such a breaking of the (magnetic) charge conjugation by the contact structure quantisation with a noncentral parmeter.

\section{Acknowledgements}

Tomasz Brzeziński would like to thank the Engineering and Physical Sciences Research Council for an Advanced Fellowship. The research of Bartosz Zieliński is supported by the EPSRC grant GR/S01078/01 


\section{References}

[1] F. Bonechi, N. Ciccoli and M. Tarlini. Noncommutative instantons and the 4-sphere from quantum groups. Comm. Math. Phys. 226 (2002), 419-432.

[2] T. Brzezinski and P. M. Hajac, Relatively projective extensions of Galois type, Swansea Preprint MRR 2001/15, (2001).

[3] T. Brzeziński, S. Majid, Quantum group gauge theory on quantum spaces, Commun. Math. Phys. 157 (1993) 591-638. Erratum 167 (1995) 235.

[4] T. Brzeziński and S. Majid, Coalgebra bundles, Comm. Math. Phys. 191 (1998), 467-492.

[5] T. Brzeziński and S. Majid, Quantum geometry of algebra factorisations and coalgebra bundles, Comm. Math. Phys. 213 (2000), 491-521.

[6] T. Brzeziński and A. Sitarz. Dirac monopoles from the Matsumoto non-commutative spheres. Swansea Preprint MRR 2002/9, (2002).

[7] D. Calow and R. Matthes, Connections on locally trivial quantum principal fibre bundles, J. Geom. Phys., J. Geom. Phys. 41 (2002) 114-165.

[8] A. Connes and M. Dubois-Violette. Noncommutative finite-dimensional manifolds. I. Spherical manifolds and related examples Comm. Math. Phys. 230 (2002), 539-579.

[9] A. Connes and G. Landi. Noncommutative manifolds, the instanton algebra and isospectral deformations. Commun. Math. Phys. 221 (2001), 141-159.

[10] J. Cuntz and D. Quillen. Algebra extensions and nonsingularity. J. Amer. Math. Soc. 8 (1995), 251289.

[11] L. Dąbrowski, The garden of quantum spheres, Preprint. Banach Center Publications (to appear).

[12] L. Dąbrowski, H. Grosse and P. M. Hajac, Strong connections and Chern-Connes pairing in the Hopf-Galois theory, Comm. Math. Phys. 220 (2001), 301-331.

[13] L. Dąbrowski, G. Landi and T. Masuda. Instantons on the quantum 4-spheres $S_{q}^{4}$. Commun. Math. Phys. 221 (2001) 161-168.

[14] Galois Type Extensions of Noncommutative Algebras. Part I: P. Schauenburg and H.-J. Schneider, Galois type extensions and Hopf algebras. Part II: T. Brzezinski and P. M. Hajac, Galois type extensions and noncommutative geometry. Banach Center Publications (to appear).

[15] P. M. Hajac, Strong connections on quantum principal bundles, Comm. Math. Phys. 182 (1996), 579-617.

[16] P. M. Hajac and S. Majid, Projective module description of the q-monopole, Comm. Math. Phys. 206 (1999), 247-264.

[17] K. Matsumoto, Non-commutative three dimensional spheres, Japan J. Math. 17 (1991) 333-356

[18] H. Omori, Y. Maeda, N. Miyazaki, A. Yoshioka, Noncommutative 3-sphere: A model of noncommutative contact algebras, J. Math. Soc. Japan 50 (1998) 915-943.

[19] H. Omori, N. Miyazaki, A. Yoshioka, Y. Maeda, Noncommutative 3-sphere as an example of noncommutative contact algebras, Banach Center Publications 40 (1997) 329-334. 